\title{
A rare case of coexistence of tuberculosis verrucosa cutis and pulmonary tuberculosis
}

\author{
Dan Huang, Shuang Jin, Wei Zhang, Xuyue Zhou, Rong Li, Kun Chen, Mei Ju
}

Department of Physiotherapy, Hospital of Dermatology, Chinese Academy of Medical Sciences and Peking Union Medical College, Nanjing, China

Adv Dermatol Allergol 2021; XXXVIII (6): 1124-1126

DOI: https://doi.org/10.5114/ada.2021.110495

A 52-year-old Chinese builder had presented with pulmonary tuberculosis for more than two decades and asymptomatic painful erythema papules involving the buttocks for one decade. Two decades ago, he was diagnosed with tuberculosis because of haemoptysis, but his tuberculosis had not been treated systematically. A decade later, he had haemoptysis again and began to develop erythema papules on his right buttock and then they spread to the contralateral and perianal areas, but he did not seek any medical treatment. One year ago, the skin lesions deteriorated rapidly and the patient felt pruritus on them and there was purulent fluid after pressing them. There was no improvement

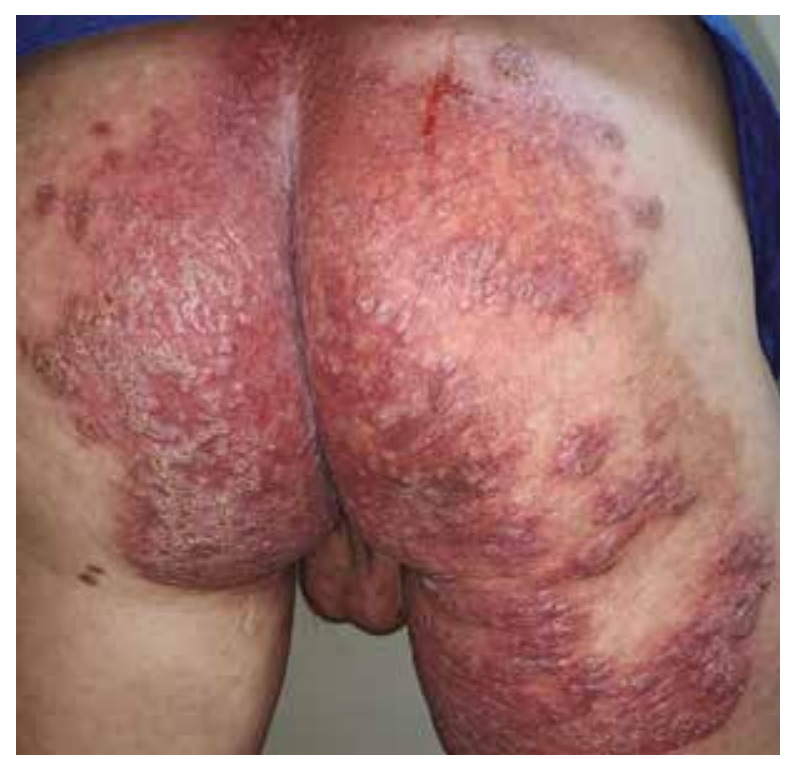

Figure 1. Clinical photos of the lesion from the patient. Multiple verrucous erythematous papules with large erythema and telangiectasia involving his perianal area, buttocks and right thigh of his condition after applying a variety of unknown ointments which the patient purchased himself. He reported no systemic symptoms such as fever, fatigue, night sweat and weight loss during the two decades. On his first visit, physical examination revealed multiple verrucous erythematous papules with large erythema and telangiectasia involving his perianal area, buttocks and right thigh lub (Figure 1). Complete blood cell count was normal. Chest radiography revealed randomly distributed miliary shadows and small nodules in both lungs, and stable calcification on the upper lobes of both lungs. A skin biopsy from the verrucous erythematous papules revealed that there was a small piece of caseation necrosis in granulomatous inflammation's central, and it was surrounded by infiltrated lymphocytes, tissue cells, and Langhans giant cells (Figure 2). PSA staining, silver staining and acid-fast staining were all negative. T-SPOT was positive. The result of DNA sequencing and bacterial culture showed the existence of Mycobacterium tuberculosis. Based on the clinical and laboratory features, a diagnosis of TCO was made. Significant improvement of the lesion was noted after two-month treatment with $75 \mathrm{mg}$ isoniazid, $150 \mathrm{mg}$ rifampicin, $400 \mathrm{mg}$ pyrazinamide and 400 mg levofloxacin, once a day. After 2 months of anti-tuberculosis treatment, his skin lesions almost disappeared (Figure 3).

Mycobacterium tuberculosis infection most commonly affects the lungs, but it also can involve extrapulmonary organs such as skin. Cutaneous tuberculosis (CTB) is a relatively uncommon form of infection accounting for $1-2 \%$ of all extrapulmonary tuberculosis (TB) cases [1]. There are four major categories of CTB [2], including inoculation tuberculosis, endogenous tuberculosis, haematogenous tuberculosis and tuberculid, and there are branches under each category, so it is difficult to diagnose due to its multiple clinical manifestations. Tuberculosis verrucosa cutis (TVC) is a rare sub-type

Address for correspondence: Kun Chen, Mei Ju, Department of Physiotherapy, Hospital of Dermatology, Chinese Academy of Medical Sciences and Peking Union Medical College, Nanjing 210042, China, phone: +86 258547 8919, fax: +86 25 85414477, e-mail: kunchen181@aliyun.com, jumeiweng@163.com Received: 1.06 .2020 , accepted: 3.11 .2020$. 
A
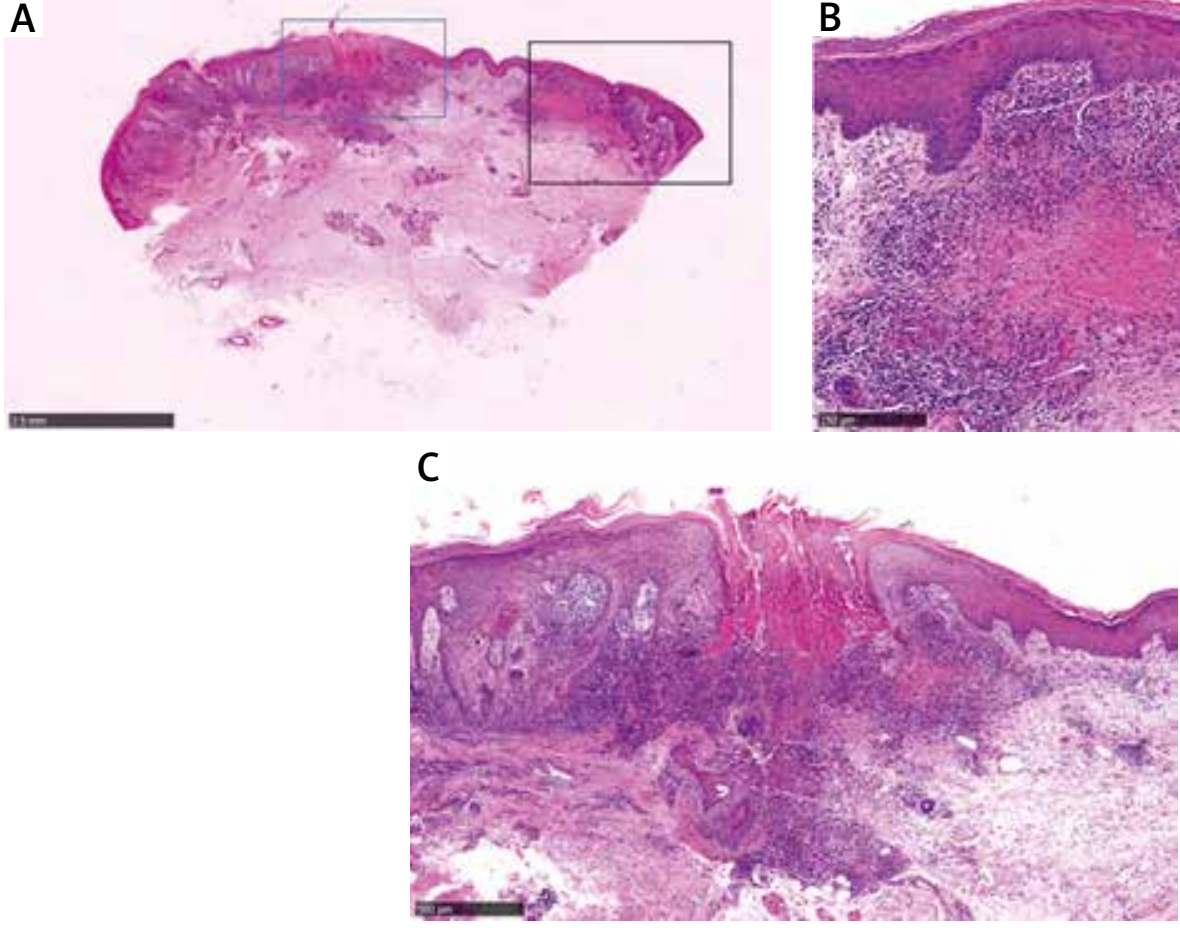

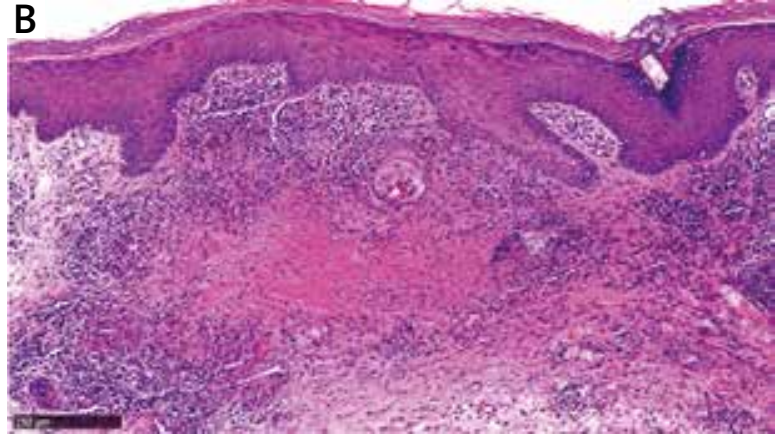

pare 2. Histopathological photos of the lesion from the patient. Histopathological examination shows hyperkeratosis, parakeratosis, acanthosis, locally ulcer and pseudoepitheliomatous hyperplasia. Neutrophil aggregation to form neutrophil microabscess in the stratum spinosum. Mixed inflammatory infiltrate composed of lymphocytes, tissue cells, and neutrophil in the superficial dermis to form granulomatous inflammation and its central small piece of caseation necrosis, surrounded by infiltration of lymphocytes, tissue cells, Langhans giant cells

of CTB, which often occurs in the buttocks and is caused by exogenous infection in previously sensitized individuals [2].

Clinically, the manifestation of TVC is non-specific which often begins from a small papule, soon becomes a hyperkeratotic verrucous plaque and usually with irregular borders [2], but it can also present as other cutaneous lesions. And the frequent sites of TVC are the dorsa of the fingers, hands, ankles and buttocks [2]. In this reported case, the skin lesion occurred on the right buttock and then spread to the perianal area which mainly showed multiple verrucous erythematous papules with large erythema and telangiectasia. And there were many necrotic nodules under the skin which had not been broken. This might be attributed to the hormone or antibiotic-contained self-applied ointment which reduced skin inflammation.

Histologically, it usually presents as pseudoepitheliomatous hyperplasia of the epidermis and hyperkeratosis, in the upper and mid-dermis, suppurativa and granulomatous inflammation can be seen [3]. To obtain an accurate diagnose, bacteria culture and PCR-based DNA sequencing are considered the best for pathological organism identification [3]. In our patient, both PCR and bacterial culture for Mycobacterium tuberculosis were positive and with his history of pulmonary tuberculosis and the clinical and laboratory features, his diagnosis was TVC. But our case should be differentiated from tuberculosis cutis orificialis which is caused by auto-inoculation Mycobacterium tuberculosis in patients with advanced pulmonary and gastrointestinal tuberculosis. This patient reported no history of bellyache or diarrhoea and the skin lesion firstly occurred on the

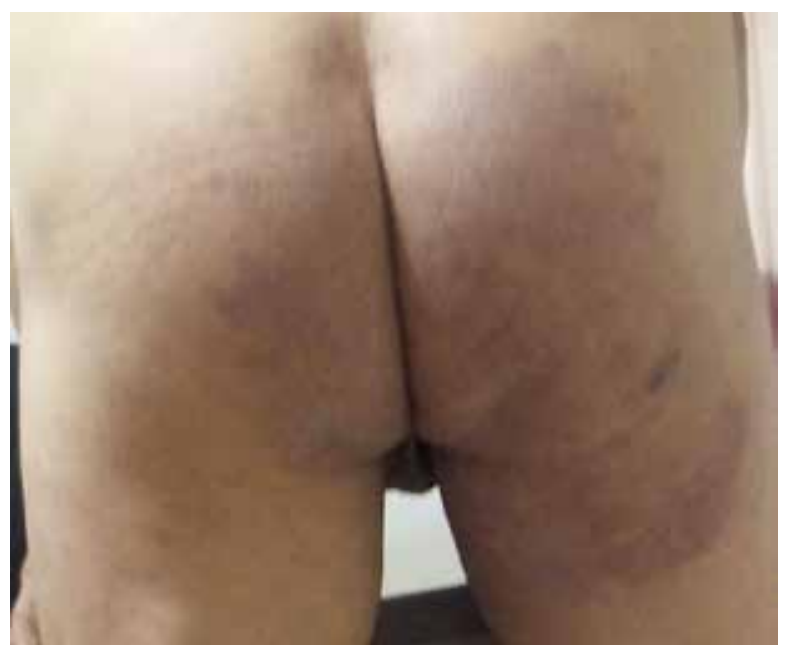

Figure 3. Clinical photos of the lesion from the patient after treatment. Completely healed lesions with post-inflammatory hyperpigmentation over the perianal area, buttocks and right thigh after treatment 
right buttock and then spread to the perianal area, so it is more likely to be TVC.

In our case, the patient had a history of pulmonary tuberculosis but reported no systemic symptoms associated with tuberculosis, except for haemoptysis. The case of TVC with active pulmonary tuberculosis is rare. There were several case reports similar to ours such as Yang's case [4] with an enlarging verrucous plaque, and Eun's case [5] with mildly pruritic hyperkeratotic erythematous plaques. Due to the non-specific manifestations of TVC, clinicians may easily overlook it. In our case, the patient who showed no systemic symptoms had a long history of disease and atypical lesions, and he bought unknown ointments which might contain hormone or antibiotics, which may change the appearance of the lesions for a long term. In summary, TVC should be considered when a patient has chronic painful perianal lesions to decrease missed diagnosis and misdiagnosis.

\section{Acknowledgments}

This work was supported by the National Natural Science Foundation of China $(81872545,81703152)$ and CAMS Innovation Fund for Medical Sciences (CIFMS2017-I2M-1-017).

Dan Huang and Shuang Jin contributed equally to this work and should be regarded as co-first authors.

\section{Conflict of interest}

The authors declare no conflict of interest.

\section{References}

1. Bravo F G, Gotuzzo E. Cutaneous tuberculosis. Clin Dermatol 2007; 25: 173-80.

2. Santos JB, Figueiredo AR, Ferraz CE, et al. Cutaneous tuberculosis: epidemiologic, etiopathogenic and clinical aspects - part I. An Bras Dermatol 2014; 89: 219-28.

3. Dias MF, Bernardes Filho F, Quaresma MV, et al. Update on cutaneous tuberculosis. An Bras Dermatol 2014; 89: 925-38.

4. Yang TT, Su YC. A rare case of tuberculosis verrucosa cutis on the buttocks. Kaohsiung J Med Sci 2020; 36: 222-3.

5. Eun DH, Kim JY, Choi YH, et al. Long-standing verrucous plaques on the buttocks: a quiz. Tuberculosis verrucosa cutis. Acta Derm Venereol 2018; 98: 161-2. 